\title{
COVID-19 en el adulto mayor: características clínicas e impacto sobre la salud mental
}

COVID-19 in the elderly: clinical features and impact on mental health

Cristian Alejandro Álvarez ${ }^{1}$ (D) https://orcid.org/0000-0002-8624-1408, Carlos Rafael Ávila1 (D https://orcid.org/0000-0002-7840-2996, Nency Yarely Garcia ${ }^{1}$ (D https://orcid.org/0000-0003-2558-1983, Wilmer Edgardo Quintanilla1 (D https://orcid.org/0000-0002-7617-9081, Manuel Antonio Sierra² ${ }^{2}$ (D) https://orcid.org/0000-0001-7684-8735.

1,2Universidad Tecnológica Centroamericana, Facultad de Ciencias de la Salud; Tegucigalpa, Honduras.

RESUMEN. El propósito de esta revisión fue analizar la literatura existente con el fin de identificar los principales aspectos de la enfermedad ocasionada por el SARS-CoV-2, así como su impacto en la salud mental del adulto mayor y su relación con la mortalidad en esta población en riesgo. Se revisó la información a partir de artículos de revistas electrónicas a través de las bases de datos Pubmed, Medline, Scopus y Google Scholar para el período julio 2020 a junio 2021. Entre los aspectos relevantes se encontró datos alarmantes, los pacientes con comorbilidades en este grupo de edad tenían 6 veces más probabilidad de ser hospitalizados y 12 veces más probabilidad de morir que una persona sin comorbilidades. La salud mental es un importante factor en esta pandemia, se ha demostrado deterioro en estado general y enfermedades mentales debido a la ansiedad y aislamiento social secundario a la pandemia en este grupo.

Palabras clave: Adulto mayor; Infecciones por Coronavirus; Salud Mental.

Recibido: 15-07-2020 Aceptado: 03-09-2021 Primera vez publicado en línea: 24-09-2021 Dirigir correspondencia a: Dr. Cristian Álvarez

Correo electrónico: cristianalvarez@unitec.edu

RELACIONES Y ACTIVIDADES FINANCIERAS Y NO FINANCIERAS: Ninguno

DECLARACIÓN DE CONFLICTOS DE INTERÉS: Ninguno

Forma de citar: Álvarez CA, Ávila CR, García NY, Quintanilla WE, Sierra MA. COVID-19 en el adulto mayor: características clínicas e impacto sobre la salud mental. Rev Méd Hondur. 2021; 89 (2): 142-147. DOl: https://doi.org/10.5377/rmh.v89i2.12367

(C) 2021 Autor(es). Artículo de acceso abierto bajo la licencia https://creativecommons.org/ licenses/by/4.0/deed.es

\section{INTRODUCCIÓN}

La enfermedad del coronavirus o COVID-19, inicialmente llamado 2019-nCoV, fue oficialmente declarada como una pandemia por la Organización Mundial de la Salud (OMS) el 11 de marzo del 2020. ${ }^{1}$ Según el último reporte del Centro para la Seguridad de Salud de Johns Hopkins, hasta el día 04 de Julio del 2021, se reportó 183,649,047 millones de casos confirmados de COVID-19 con aproximadamente 3,973,892 millones muertes. Con un promedio de 375,056 casos detectados por semana a nivel mundial. ${ }^{2}$

La principal forma de transmisión del virus es de persona a persona, ya que cuando una persona tose o estornuda, lleva una carga viral importante en sus secreciones que pueden existir como aerosoles hasta por 3 horas y pueden permanecer en ciertas superficies hasta por 72 horas. $^{3}$

En reportes nacionales la edad promedio de los casos reportados es igual o mayor a los 60 años tanto en hombres como en mujeres. También se les asocia con un mayor porcentaje de mortalidad en comparación a la población joven, por ende, la importancia de enfocarnos en este grupo etario. ${ }^{4}$ Además, se determinó que los pacientes adultos mayores con comorbilidades comunes como la enfermedad cardiovascular, diabetes, Enfermedad Pulmonar Obstructiva Crónica (EPOC) o enfermedades neoplásicas tenían 6 veces más probabilidad de ser hospitalizados y 12 veces más probabilidad de morir que una persona sin comorbilidades. Así mismo, 8 de cada 10 pacientes que mueren por COVID-19 en Estados Unidos, son adultos mayores. ${ }^{5}$

En las personas mayores de 60 años existe una importante preocupación ya que, el confinamiento prolongado, la ansiedad por la pandemia, y el sentimiento de soledad podría empeorar el estado de salud mental. ${ }^{1}$ El aislamiento social y la sensación de soledad en este grupo ha mostrado estar directamente relacionado a síntomas de depresión $(p<0.0001)$ y síntomas de ansiedad $(p<0.0001){ }^{5}$

En Honduras, se reportó 266,918 casos confirmados, concentrándose sobre todo en los departamentos de Francisco Morazán y Cortés $(27.5 \%$ y $25.4 \%$ del total de casos respectivamente), con un total de 7,081 muertes confirmadas por CO- 
VID-19, esto según la página oficial del gobierno actualizada al 04 de Julio del $2021 .{ }^{6}$

Se realizó una búsqueda exhaustiva en diferentes buscadores científicos Pubmed, Medline, Scopus, Google Scholar, desde el 01 de julio del 2020 hasta el 04 de Julio del 2021 en busca de referencias sobre el tema de interés, la pandemia de la COVID-19 y su relación a los pacientes adultos mayores. Las palabras claves utilizadas incluían: "Infecciones por Coronavirus" "Adulto mayor" "Salud Mental". Se revisaron los artículos relevantes al tema, incluyendo artículos en inglés y español. El propósito de esta revisión fue analizar la literatura existente con el fin de identificar los principales aspectos de la enfermedad ocasionada por el SARS-CoV-2 y su impacto en la salud mental en el adulto mayor.

\section{FACTORES DE RIESGO}

Existen múltiples factores de riesgo que pueden determinar la gravedad de la infección por COVID-19. Se ha reportado que la presentación clínica depende tanto de factores genéticos tales como polimorfismo en los genes Enzima Convertidora de Angiotensina II (ECA2) y Serina-Proteasas Transmembrana de Tipo II (TMPRSS) y no genéticos como ser la edad avanzada, género masculino, y la presencia de comorbilidades. ${ }^{7}$ Muchas investigaciones han basado sus estudios en las formas graves de COVID-19 y la presencia de comorbilidades como principal factor de riesgo. Entre estas tenemos a las personas con hipertensión arterial, diabetes mellitus y enfermedad renal crónica. De acuerdo con un metaanálisis, la enfermedad renal crónica, la hipertensión arterial y la diabetes mellitus se asocian a una manifestación clínica grave en paciente con COVID-19, con incremento de más de 3.5 veces en el riesgo. ${ }^{7}$ Según la ONU en su informe "El Impacto de la COVID-19 en las Personas Mayores", el adulto mayor tiene mayor probabilidad de presentar las formas graves de la enfermedad, siendo los mayores de 80 años la población más afectada, falleciendo a una tasa 5 veces mayor que la media, y esto se debe a que según la ONU, el $66 \%$ de los adultos mayores padecen de comorbilidades. ${ }^{8}$

La obesidad es un factor de riesgo para la morbilidad y mortalidad prematura. ${ }^{7}$ Existen publicaciones recientes en relación con la pandemia actual que sugieren que las personas con obesidad tienen mayor riesgo de desarrollar una enfermedad más grave por coronavirus. Los pacientes con índice de masa corporal (IMC) mayor o igual a 35 requieren con más frecuencia de ventilación mecánica invasiva. ${ }^{9}$ En un estudio realizado por Goncalves y colaboradores en 2020, en el que se estudió un total de 176 pacientes adultos mayores con diagnóstico de COVID-19 que estuvieron en una unidad de cuidados intensivos, concluyó que el $68.7 \%$ de los pacientes tenía un índice de masa corporal mayor de 30 , y el $15 \%$ de los pacientes tenían un índice de masa corporal mayor de $35 .^{10}$

Un estudio retrospectivo publicado por Chang y colaboradores en 2020 que incluyo 211 pacientes con diagnóstico de diabetes mellitus tipo 2, quienes se encontraban asintomáticos o con sintomatología leve para COVID-19, concluyeron que este parámetro mencionado anteriormente (diabetes mellitus tipo 2) era de gran utilidad para tomar en cuenta y poner más cuidado en estos pacientes ya que son factores predictivos para el curso de la enfermedad. ${ }^{11}$ En cuanto a los adultos mayores, un estudio realizado por Zhang y Colaboradores en 2020, en el que se incluyó a un total de 131 pacientes (50 con diabetes mellitus tipo 2) se concluyó que experimentaron una neumonía más severa y disfunción multiorgánica a diferencia de los pacientes que no padecían de diabetes mellitus tipo $2(p<0.05$ o $p<0.01$ ), lo que lleva a la diabetes mellitus tipo 2 a ser un factor de riesgo independiente para la progresión a formas graves de la COVID 19. ${ }^{12}$

\section{MANIFESTACIONES CLINICAS EN EL ADULTO MAYOR}

Para comenzar a describir las manifestaciones clínicas que se presentan en esta enfermedad debemos definir período de incubación. Este se entiende como el intervalo encontrado entre la posible fecha más temprana de contacto de la fuente de transmisión y la fecha más temprana de inicio del síntoma.

Un estudio realizado en dos hospitales de la ciudad de Nueva York reportó como síntoma más común la tos $79.4 \%$, seguido de fiebre $77.1 \%$, disnea $56.5 \%$, mialgias $23.8 \%$, diarrea $23.7 \%$ y nauseas/vómitos $19.1 \%$. Cabe mencionar que dicho estudio se realizó en una muestra de 393 pacientes adultos mayores con un promedio de edad de 62.2 años. ${ }^{13}$

Es frecuente que los adultos mayores presenten síntomas difusos y poco específicos en distintas infecciones, lo que dificulta el diagnóstico. Síntomas como deterioro del estado funcional basal, caídas, delirium, deshidratación e hiporexia son frecuentes como indicadores de infecciones en esta población. En el adulto mayor se describe una disminución de la respuesta febril ante agentes infecciosos. Con los termómetros habituales entre el 20 y el $30 \%$ de los pacientes ancianos con infecciones virales o bacterianas importantes no muestran fiebre, incluso cuando presentan cuadros clínicos serios. ${ }^{13}$ Las características típicas de los pacientes adultos mayores son pérdida involuntaria de peso, alteraciones cognitivas, movilidad reducida y necesidad de asistencia en las actividades de la vida diaria. Las escalas de fragilidad son una ayuda sencilla para la clasificación de estos pacientes tanto para pronóstico como para tomar decisiones de admisión a las salas de emergencias y de cuidados intensivos. ${ }^{13}$

\section{SALUD MENTAL}

Existe preocupación, ya que el confinamiento prolongado, la ansiedad por la pandemia, y el sentimiento de soledad podría empeorar el estado de salud mental de los pacientes adultos mayores. ${ }^{14} \mathrm{El}$ envejecimiento trae consigo vulnerabilidad psicológica social aumentando el riesgo de padecer infecciones debido a la disminución de la respuesta inmune.

Muchos ancianos viven en instituciones lo cual incrementa su riesgo de vivir en hacinamiento, falta de higiene y falta de supervisión adecuada. ${ }^{14}$ Los adultos mayores de 65 años experimentan un impacto psicosocial significativo. La ansiedad, pánico, trastornos de adaptación, depresión, estrés crónico e insomnio son solo algunos de los principales efectos del aislamiento.

La desinformación y la incertidumbre dan lugar a la histeria colectiva. Entre ellos, los adultos mayores quienes están sometidos a un estrés adicional ya que son conscientes del riesgo de una mayor severidad y fatalidad al contraer el virus, compara- 
dos a otros grupos poblacionales por lo cual son especialmente vulnerables. ${ }^{14}$

El aislamiento social, aunque es una estrategia importante para luchar contra el COVID-19, también es una causa importante de soledad, particularmente en entornos como instituciones, considerado como un factor de riesgo para la depresión, trastornos de ansiedad y el suicidio. En general, los mayores de 65 años pueden presentar un aumento de niveles de ansiedad $31.8 \%$, depresión $27.5 \%$, trastorno obsesivo compulsivo $25.1 \%$ y hostilidad $13.7 \%$ durante la cuarentena. ${ }^{14}$ Un estudio realizado en la población de China, revelo que más de la mitad de la población geriátrica experimentaba estrés psicológico de moderado a severo secundario a la pandemia. ${ }^{15}$

En su trabajo, Gutiérrez recalca que la OMS sugiere a cuidadores de adultos mayores de 65 años aumentar el acompañamiento y el apoyo emocional. Es importante brindar la información sobre la enfermedad con datos sencillos acerca de lo que está pasando y las formas de disminuir el riesgo de infección de forma tal que lo puedan entender. ${ }^{14}$

El seguimiento de la salud mental de la población se debe continuar por lo menos 6 meses después de finalizado el confinamiento. Se estima que la prevalencia de síntomas de estrés postraumático en la población general pasó del $4 \%$ al $41 \%$ y la prevalencia de depresión aumentó un $7 \% .{ }^{14}$

\section{DIAGNÓSTICO}

El diagnóstico final de la enfermedad recae en la utilización de la Reacción en Cadena Polimerasa en tiempo real (rT-PCR), y en los estudios de imagen que se han vuelto de suma importancia para la evaluación de la enfermedad. ${ }^{16} \mathrm{Un}$ estudio publicado por Wang y colaboradores, demostró la efectividad de la rT-PCR, según el sitio de la muestra, se reportó un porcentaje de detección del virus mayor del $90 \%$ en muestras de lavado bronco alveolar, el $90 \%$ en saliva, y un $70 \%$ en esputo y en los hisopados nasofaríngeos. ${ }^{17}$

En cuanto a estudios como el hemograma y la química sanguínea, Neumann-Podczaska y colaboradores reportan que en el adulto mayor se observó linfopenia en una cantidad considerable de estudios, al igual que trombocitopenia ocasional. Además, la proteína $\mathrm{C}$ reactiva, velocidad de eritrosedimentacion globular (VSG) estaban evidentemente elevadas en los pacientes, y la mayoría informó niveles $>10 \mathrm{mg} / \mathrm{L}$ y $>35 \mathrm{~mm} / \mathrm{h}$ respectivamente. Pocos estudios también informaron niveles más altos de $\mathrm{LDH}$ (> $300 \mathrm{U} / \mathrm{L}$ ) y dímeros $\mathrm{D}(>1.0 \mathrm{mg} / \mathrm{L})$. Los marcadores hepáticos y cardíacos estuvieron principalmente dentro del rango normal, mientras que algunos estudios mostraron marcadores renales levemente anormales. ${ }^{18}$

Un estudio publicado por Vakili y colaboradores en 2020, encontró que la proporción de linfocitos en el grupo de adultos mayores fue significativamente menor que en el grupo de jóvenes y de mediana edad $(p<0.001)$, y la proteína $C$ reactiva fue significativamente mayor que en el grupo de jóvenes y de mediana edad $(p<0.001)$. No hubo diferencias significativas en el recuento de glóbulos blancos, proporción de neutrófilos, procalcitonina, nivel de hemoglobina, plaquetas y creatinina sérica en los dos grupos. ${ }^{19}$
Las radiografías de tórax no muestran ninguna alteración en pacientes con sintomatología leve o asintomáticos, sin embargo, se observó que en pacientes que requerían hospitalización, el $69 \%$ tuvo hallazgos radiológicos anormales a su ingreso y el $80 \%$ mostro anormalidades durante su estancia hospitalaria, siendo estas anormalidades más extensas a las 2 semanas de iniciada la sintomatología. ${ }^{20}$ La descripción más común reportada en el adulto mayor fueron opacidades en vidrio esmerilado $28.6 \%$ o consolidaciones $12.9 \%$, que afectan a múltiples lóbulos $62.2 \%$ en una distribución bilateral $58.2 \%$. Se encontró derrame pleural en un $3.7 \%$ de los pacientes y hallazgos radiológicos normales en $2.7 \%{ }^{18}$

La tomografía computarizada (TC) de tórax es muy importante en el diagnóstico complementario del nuevo tipo de neumonía, y los cambios en la TC son variables. Dependiendo del curso de la enfermedad, la neumonía puede involucrar uno o más lóbulos simultáneamente, siendo las opacidades en vidrio esmerilado el hallazgo tomográfico más importante en este tipo de pacientes. ${ }^{21}$

Para los pacientes adultos mayores, la relación entre la línea subpleural y el grosor pleural fue mayor que la de los pacientes jóvenes debido al deterioro de la estructura y función pulmonar y la baja función inmune del cuerpo, se redujo la distensibilidad pulmonar. Esto puede indicar que los pacientes de edad avanzada muestran una respuesta inflamatoria más grave. Al mismo tiempo, en los adultos mayores, los lóbulos pulmonares completos eran más propensos a estar afectados. Esto puede indicar que, en el mismo curso de la enfermedad, la progresión de la enfermedad en los adultos mayores fue más rápida que en los pacientes jóvenes. ${ }^{22}$

\section{DIAGNÓSTICOS DIFERENCIALES}

Uno de los principales desafíos médicos en cuanto al SARS-CoV-2, es la sintomatología inespecífica que presenta, al igual que los hallazgos radiológicos, lo cual ocasiona un problema al momento de realizar un diagnóstico certero y definitivo debido a su similitud a otros virus respiratorios que circulan en nuestro entorno, por lo que es de suma importancia realizar un diagnóstico diferencial con las otras patologías para que de esa forma se trate oportunamente al paciente.

Las neumonías virales ya sea causadas por adenovirus, influenza, virus sincitial respiratorio y otros, se diferencian del COVID-19 en algunas características, en cuanto al patógeno, el COVID-19 es causado por el SARS-CoV-2, y en un inicio existió una historia de exposición al virus en áreas de transmisión. En cuanto al diagnóstico laboratorial y de imagen las demás neumonías virales encontramos un rT-PCR positivo para dicho patógeno, linfocitosis, inflamación intersticial y un patrón reticular mientras que en el COVID-19, tendríamos rT-PCR positivo para SARS-CoV-2, linfopenia, y patrón en vidrio esmerilado a nivel de la tomografía computarizada. ${ }^{23,24} \mathrm{En}$ las técnicas de imagen, se presenta más que todo los consolidados neumónicos y lóbulos centronodulares. ${ }^{23,24}$

Durante la pandemia de COVID-19, el diagnóstico diferencial es crucial para los adultos mayores. Gran parte de la neumonía viral con patrones radiológicos intersticiales puede deberse a muchos virus diferentes (neumonía de influenza A y 
B, parainfluenza y coronavirus, micoplasma) y muchos modelos clínicos y radiológicos son en realidad mixtos (neumonía bacteriana y viral) en este grupo poblacional. Las neumonías de origen bacteriano también muestran algunas diferencias clínicas y diagnosticas en cuanto al COVID-19, estas son más comunes en el invierno. La tos productiva y fiebres altas son síntomas predominantes, mientras que la obstrucción nasal, la rinorrea, y la odinofagia son síntomas leves, no muy frecuentes. Laboratorialmente, encontramos leucocitosis, la proteína c-reactiva y Velocidad de Eritrosedimentación. ${ }^{25}$

\section{TRATAMIENTO}

La evidencia disponible sobre tratamientos farmacológicos para poder controlar la infección por COVID-19 es bastante limitada y de muy baja calidad debido a que se trata de una nueva entidad totalmente desconocida. A medida que el tiempo avanza y la pandemia continúa acechando en los diferentes países, afectando unos países más que a otros, se ha podido aprender un poco más acerca del manejo y abordaje de dicho virus. Es muy claro que las experiencias vividas en países tales como China, Italia, Estados Unidos entre otros, han sido de mucha ayuda, que a través de prueba y error se ha logrado conocer y se ha podido mejorar cada día más en cuanto al manejo tanto de los pacientes críticos y no críticos afectados por el COVID-19. ${ }^{26}$

La oxigenoterapia en la actualidad continúa siendo el pilar del tratamiento para los pacientes con COVID-19 que requieren hospitalización. Más del $75 \%$ de los pacientes hospitalizados con COVID-19 requieren oxigenoterapia suplementaria. Para pacientes que no responden a la terapia de oxígeno convencional, se recomienda oxígeno de cánula nasal de alto flujo o en su defecto, ventilación mecánica invasiva. ${ }^{26,27}$

En la actualidad no existe ningún medicamento aprobado por la Administración de Medicamentos y Alimentos (FDA por sus siglas en inglés) que funcione en un cien por ciento para tratar a una persona con infección por coronavirus.

La ivermectina, una droga antiparasitaria que en los últimos años ha mostrado efectos antivirales in vitro, y se ha estudiado para el tratamiento del SARS-CoV-2. En un estudio realizado por Formiga y colaboradores en el año 2020 en Australia, se demostró que la ivermectina tiene una actividad antiviral de amplio espectro in vitro, contra el virus SARS-CoV-2 capaz de efectuar una reducción del ARN viral a las 48 horas de la infección por el virus. ${ }^{29}$ Así mismo, nos menciona esta droga antiparasitaria no ha sido estudiada en células pulmonares, lo cual es un aspecto de suma importancia en el SARS-CoV-2. Sin dejar de mencionar que es muy probable que para alcanzar concentraciones inhibitorias se necesitaría de regímenes de dosis muy elevadas en humanos, lo que podría conllevar a efectos secundarios no deseados. $^{28}$

Los efectos terapéuticos de la dexametasona han sido muy estudiados en esta pandemia. En un artículo original publicado en el New England Journal of Medicine, se encontró que la dexametasona intravenosa reducía significativamente la mortalidad en los pacientes con COVID-19 que estaban recibiendo soporte ventilatorio o ventilación mecánica, pero no redujo la mortalidad de los pacientes que no requerían soporte ventilatorio. ${ }^{29,30}$
La incidencia de enfermedad tromboembólica venosa en pacientes con COVID-19 en unidades de cuidados intensivos es mayor en comparación con lo reportado en otras enfermedades. En series internacionales se ha demostrado en términos histopatológicos la existencia de trombosis microvascular y hemorragia pulmonar, por lo que la anticoagulación profiláctica se ha usado como parte del tratamiento de enfermos críticos que tienen concentraciones elevadas de dímero $\mathrm{D}$, alteraciones en los parámetros de coagulación, coagulación intravascular diseminada, síndrome de liberación de citocinas o insuficiencia orgánica múltiple. ${ }^{31}$

Los adultos mayores suelen estar polimedicados y deben evaluarse las interacciones medicamentosas ante los probables tratamientos a efectuarse para minimizar los efectos adversos. También debe monitorizarse estrechamente la función renal. La incidencia de lesión renal aguda en pacientes ingresados con COVID-19 aún no está bien establecida, pero oscila del $0.1 \%$ al $29 \%$. El desarrollo de lesión renal aguda está asociada a un aumento de la mortalidad que puede llegar al $91 \%$ en caso de Distrés Respiratorio Agudo (SDRA) con lesión renal aguda. ${ }^{18}$

\section{PREVENCIÓN}

La prevención es una de las medidas más importantes en esta patología, así como cualquier otra. Sin embargo, debido a su alto riesgo de contagio las medidas recomendadas de prevención suelen ser un poco más estrictas. Entre las medidas preventivas de bioseguridad que se han establecido se encuentra el desinfectar las superficies y manos con el adecuado lavado frecuente y uso de gel antibacterial. ${ }^{32}$

El uso de equipo de protección personal (mascarilla, guantes de latex, lentes de protección ocular, careta facial, bata quirúrgica) se ha convertido en norma universal en la mayoría de los países del mundo durante esta pandemia declarada por la OMS. $^{32}$

Según el Centro Nacional para la Información Biotecnológica (NCBI) en un artículo publicado en julio 2020, indicó que es importante diferenciar entre aislamiento, cuarentena y distanciamiento físico. Respecto al término cuarentena esta hace referencia a la restricción voluntaria u obligatoria del desplazamiento de individuos que han estado expuestos a un potencial contagio y que posiblemente se encuentren infectados. El aislamiento se refiere a la separación física de las personas contagiadas de aquellas que están sanas. Mientras que el distanciamiento físico consiste en alejarse de lugares concurridos y restringir la interacción entre las personas tomando cierta distancia física o evitando el contacto entre ellas. ${ }^{33}$

Las medidas preventivas se basan principalmente en cumplir con aislamiento. Esta se puede cumplir dentro de un hospital en caso de que se encuentre infectado, con criterios de hospitalización. En los casos con síntomas leves o asintomáticos el paciente debe cumplir con aislamiento o cuarentena dentro de su casa conocido como autoaislamiento. Entre otras medidas existe el distanciamiento físico, el cual recomienda mantenerse distanciado por lo menos 2 metros entre personas. La distancia personal se encuentra estrictamente establecida entre las normas para la prevención del contagio. ${ }^{32}$ Entre otras 
se encuentran: limitar la cantidad de visitantes en establecimientos comerciales, evitar aglomeraciones y/o reuniones de gran cantidad de personas, medidas estrictas de cuarentena en zonas/edificios residenciales, quedarse en casa y salir solo si es estrictamente necesario, toma temperatura constante con termómetro infrarrojo.

Desde el punto de vista de la salud mental en el adulto mayor, es necesario implementar medidas para evitar o disminuir los daños causados por la depresión y ansiedad en estos grupos de riesgo, como la tecnología que nos conecta con familiares, amigos y profesionales de salud; contribuyendo a disminuir los síntomas mentales en el adulto mayor mediante atenciones médicas y terapias virtuales. ${ }^{14}$

Existen otras medidas como el estilo de vida saludable, prácticas de Yoga y Taichi que mejoran el afrontamiento al estrés y que mejoran el bienestar físico, ayudando a pacientes con endocrinopatías, enfermedades cardiorrespiratorias y osteomusculares; así mismo ayuda a controlar los trastornos de sueño, ansiedad y depresión. ${ }^{14}$

\section{CONCLUSIÓN}

Se debe prestar especial atención al adulto mayor durante esta pandemia ya que tras el contagio por el SARS-CoV-2 corren un mayor riesgo de hospitalización y mortalidad. El adulto mayor tiene mayor probabilidad de presentar las formas graves de la enfermedad, siendo los mayores de 80 años la población más afectada. ${ }^{8}$

Es importante considerar que los adultos mayores se pueden presentar con síntomas atípicos de la enfermedad causada por el SARS-CoV-2, lo que dificulta el diagnóstico de la enfermedad. La hiporexia, deshidratación y la hipoxia son factores importantes para tomar en cuenta en la clínica de este tipo de pacientes.

Los adultos mayores y más jóvenes con enfermedad por coronavirus tienen algunas características comunes de la tomografía computarizada, pero es más probable que los pacientes de edad avanzada tengan una afectación extensa del lóbulo pulmonar y una línea subpleural y un engrosamiento pleural. Estas características diferenciadas pueden estar relacionadas con la evolución y pronóstico de la enfermedad. ${ }^{22}$ Aun no existe un tratamiento farmacológico especifico aprobado por la FDA que demuestre su eficacia ante la infección grave del adulto mayor por el COVID-19. ${ }^{26}$

Múltiples estudios muestran que la salud mental de los pacientes adultos mayores es afectada no solo por la ansiedad que genera la posibilidad de que ellos o sus seres queridos se contagien, sino también las consecuencias del aislamiento. Siendo necesario implementar medidas para evitar o disminuir los daños causados por la depresión y ansiedad, como uso de la tecnología para la comunicación, estilos de vida saludable, con el fin de mejorar el afrontamiento al estrés y el bienestar físico.

Los sistemas de salud deben enfocarse en la equidad y en alcanzar los grupos de riesgo como los pacientes de la tercera edad, quien se ha demostrado tienen mayor probabilidad de hospitalización y mortalidad por este virus, pero no siempre son priorizados al momento de invertir recursos de salud.

\section{CONTRIBUCIONES}

Todos los autores participaron en la concepción de la idea original del artículo, en la búsqueda bibliográfica y redacción de este. MS lideró la conducción de la investigación. CA, WQ, lideraron la redacción del artículo e incorporaron las recomendaciones editoriales. Todos los autores aprobaron la versión final del artículo.

\section{DETALLES DE AUTOR(ES)}

Cristian Alejandro Álvarez, Médico General; cristianalvarez@ unitec.edu

Carlos Rafael Ávila, Médico General; carlos.avila@unitec.edu Nency Yarely Garcia, Médica General; nencygarcia@unitec.edu Wilmer Edgardo Quintanilla, Médico General; wquintanilla@ unitec.edu Manuel Antonio Sierra, Médico PhD; mass_honduras_2006@ yahoo.com

\section{REFERENCIAS}

1. World Health Organization. Coronavirus disease (COVID-19) Situation Report 155. [Internet]. Washington D.C.: WHO; 2020. [consultado 12 agosto 2020]. Disponible en: https://bit.ly/2PjNizJ

2. Johns Hopkins Center for Health Security. COVID-19 situation report june 22, 2020. [Internet]. Baltimore:John Hopkins Bloomberg School of Public Health; 2020. [consultado 20 agosto 2020] Disponible en: https://myemail. constantcontact.com/COVID-19-Updates---June-22.html?soid=1107826135 286\&aid=Ssz8NKClfLk

3. Van Doremalen N, Morris DH, Holbrook MG, Gamble A, Williamson BN, Tamin A, et al. Aerosol and surface stability of SARS-CoV-2 as compared with SARS-CoV-1. N Engl J Med [Internet]. 2020 [consultado 12 agosto 2020]; 382:1564-1567.

4. Red Nacional de Vigilancia Epidemiológica (ES). Análisis de los casos de COVID-19 notificados a la RENAVE hasta el 10 de mayo en España. [Internet]. España: RENAVE; 2020. [consultado 12 agosto 2020] Disponible en: https://bit.ly/3deKPPd

5. Center for Disease Control and Prevention. Severe outcomes among patients with coronavirus disease 2019 (COVID-19) United States, february 12- march 16, 2020. MMWR Morb Mortal Wkly Rep [Internet]. 2020 [consultado 12 agosto 2020]; 69:369-346. Disponible en: https://www.cdc. $\mathrm{gov} / \mathrm{mmwr} / \mathrm{volumes} / 69 / \mathrm{wr} / \mathrm{mm} 6912 \mathrm{e} 2 . \mathrm{htm}$

6. Despacho de Comunicaciones y Estrategia Presidencial (HN). Coronavirus COVID-19 en Honduras. [Internet]. Tegucigalpa: DCEP; 2020. [consultado 14 septiembre 2020]. Disponible en: https://covid19honduras.org/

7. Wang C, Pan R, Wan X, Tan Y, Xu L, Ho CS, et al. Immediate psychological responses and associated factors during the initial stage of the 2019 Coronavirus Disease (COVID-19) epidemic among the general population in China. Int J Environ Res Public Health [Internet]. 2020 [consultado 12 agosto 2020];17(5):1729. Disponible en: https://pubmed.ncbi.nlm.nih. gov/32155789/

8. Organización Panamericana de la Salud, Organización Mundial de la Salud. Las personas mayores de 60 años han sido las más afectadas por la COVID-19 en las Américas. Washington D.C.: OPS/OMS; 2020 [consultado 10 octubre 2020] Disponible en: https://www.paho.org/es/noticias/30-92020-personas-mayores-60-anos-han-sido-mas-afectadas-por-covid-19americas

9. Petrova $D$, Salamanca Fernández $E$, Rodriguez Barranco $M$, Navarro Pérez P, Jiménez Moleon JJ, Sánchez MJ, et al. La Obesidad como 
factor de riesgo en personas con COVID-19: posibles mecanismos e implicaciones. Atención Primaria [Internet]. 2020 [consultado 11 septiembre 2020];52(7):496-500. Disponible en: https://www.sciencedirect.com/science/ article/pii/S0212656720301657

10. Gonçalves TJM, Gonçalves SEAB, Guarnieri A, Risegato RC, Guimarães MP, de Freitas DC, et al. Prevalence of obesity and hypovitaminosis $D$ in elderly with severe acute respiratory syndrome coronavirus 2 (SARS-CoV-2). Clin Nutr ESPEN [Internet]. 2020 [consultado 25 agosto 2020]40:110-114. Disponible en: https://www.ncbi.nlm.nih.gov/pmc/articles/PMC7552968/

11. Thais M, Plasencia-Urizarri R, Aguilera-Rodríguez LE, Almaguer-Mederos. Hipertensión, diabetes y enfermedad renal crónica como factores de riesgo para Covid-19 [Internet]. Primer Congreso Virtual de Ciencias Básicas Biomédicas en Granma. Manzanillo; 2020. [consultado 25 agosto 2020]. Disponible en: http://cibamanz2020.sld.cu/index.php/cibamanz/ cibamanz2020/paper/view/496/267

12. Zhang P, Wang M, Wang Y, Wang Y, Li T, Zeng J, Wang L, Li C, Gong Y. Risk factors associated with the progression of COVID-19 in elderly diabetes patients. Diabetes Res Clin Pract [Internet]. 2021 [consultado 25 agosto 2020];171:108550. Disponible en: https://doi.org/10.1016/j. diabres.2020.108550

13. Rozenek S, Soengas N, Giber F, Murgieri M. COVID-19 en adultos mayores: ¿corresponde usar la misma definición de caso? Rev. Arg. Gerontol Geriatr [Internet]. 2020 [consultado 12 agosto 2020];34(1):3-6. Disponible en: http:// www.sagg.org.ar/wp/wp-content/uploads/2020/04/RAGG_04_2020-3-6.pdf

14. Gutiérrez Babativa L. Salud mental en adultos mayores relacionada al aislamiento por Covid-19. [Internet]. Colombia: Universidad de los Andes; 2020. [consultado 25 febrero 2021]. Disponible en: https://medicina. uniandes.edu.co/es/articulos- publicados/salud-mental-relacionada-alaislamiento-covid-19

15. Ribot Reyes VC, Chang Paredes N, Gonzalo Castillo AL. Efectos de la COVID-19 en la salud mental de la población. Rev haban cienc méd [Internet]. 2020 [consultado 25 febrero 2021];19(sup.1): e3307. Disponible en: http://scielo.sld.cu/scielo.php?script=sci_arttext\&pid=\$1729519X2020000400008

16. Long $\mathrm{C}, \mathrm{Xu} \mathrm{H}$, Shen $\mathrm{Q}$, Zhang $\mathrm{X}$, Fan $\mathrm{B}$, Wuan $\mathrm{C}$, et al. Diagnosis of the Coronavirus disease (COVID-19): rRT-PCR or CT? Eur J Radiol [Internet]. 2020 [consultado 12 agosto 2020];126:108961. Disponible en: https:// pubmed.ncbi.nlm.nih.gov/32229322/ agosto 2020];126:108961.

17. To KK, Tsang OT, Leung WS, Tam AR, Wu TC, Lung DC, et al. Temporal profiles of viral load in posterior oropharyngeal saliva samples and serum antibody responses during infection by SARS-CoV-2: an observational cohort study. Lancet Infect Dis [Internet]. 2020 [consultado 12 agosto 2020];20(5):565-574. Disponible en: https://pubmed.ncbi.nlm.nih. gov/32213337/

18. Neumann-Podczaska A, Al-Saad SR, Karbowski LM, Chojnicki M, Tobis S, Wieczorowska-Tobis K. COVID 19 - Clinical Picture in the Elderly Population: A Qualitative Systematic Review. Aging Dis [Internet].. 2020 [Consultado 12 agosto 2020];11(4):988-1008. do Disponible en: https://www.ncbi.nlm.nih. gov/pmc/articles/PMC7390523/

19. Vakili S, Savardashtaki A, Jamalnia S, Tabrizi R, Nematollahi MH, Jafarinia $M$, et al. Laboratory Findings of COVID-19 Infection are Conflicting in Different Age Groups and Pregnant Women: A Literature Review. Arch Med Res [Internet]. 2020.[consultado 12 de agosto 2020];51(7):603-607] Disponible en: https://doi.org/10.1016/j.arcmed.2020.06.007

20. Sarkodie B, Kwadwo OP, Brakohiapa E. Diagnosing COVID-19 from Chest $X$-ray in Resource Limited Environment-Case Report. Med Case Rep. [Internet] 2020 [consultado 12 agosto 2020];6(2):135. Disponible en: https:// medical-case-reports.imedpub.com/diagnosing-covid19-from-chest-xray-inresource-limited-environmentcase-report.php?aid=26888

21. Dai WC, Zhang Hw, Yu J, Xu HJ, Chen H, Luo Sp, et al. CT Imaging and differential diagnosis of COVID-19. Can Assoc Radiol J [Internet]. 2020 [consultado 13 Agosto 2020];71(2):195-200. Disponible en: https://pubmed. ncbi.nlm.nih.gov/32129670/

22. Zhu T, Wang Y, Zhou S, Zhang N, Xia L. A Comparative Study of Chest Computed Tomography Features in Young and Older Adults With Corona Virus Disease (COVID-19). J Thorac Imaging [Internet]. 2020. [consultado el 12 de agosto 2020];35(4):W97-W101. Disponible en: https://doi.org/10.1097/ rti. 0000000000000513

23. Sharma R, Agarwal M, Gupta M, Somendra S, Saxena SK. Clinical Characteristics and Differential Clinical Diagnosis of Novel Coronavirus
Disease 2019 (COVID-19). Coronavirus Disease 2019 (COVID-19) [Internet]. 202 0[consultado 25 septiembre 2020];30:55-70. Disponible en: https://www.ncbi.nlm.nih.gov/pmc/articles/PMC7189401/

24. Lei P, Fang B, Wang P. Differential diagnosis for Coronavirus Disease (COVID-19): beyond radiologic features. Am J Roentgenol [Internet]. 2020 [consultado 25 septiembre 2020];215(1):w19. Disponible en: https:// pubmed.ncbi.nlm.nih.gov/32239972/

25. de Blasio A, Chioni L, Adorn, G. Differential Diagnosis of Types of Pneumonia in the Elderly. In: Covid-19 airway management and ventilation strategy for critically ill older patients [Internet]. Berlin: Springer; 2020: 35-66. [consultado 21 agosto 2020] Disponible en: https://doi.org/10.1007/978-3030-55621-1 5

26. Berlin DA, Gulick RM, Martínez FJ. Severe Covid-19. N Engl J Med [Internet]. 2020 [consultado 21 agosto 2020];383:2451-2460. Disponible en: https://www.nejm.org/doi/full/10.1056/NEJMcp2009575

27. Vélez M, Vélez V, Marín IC, Castaño D, Velásquez Salazar P, Vera Giraldo $\mathrm{CY}$, et al. Tratamiento farmacológico de la infección COVID-19 en adultos [Internet]. Antioquia(CL): UNED; 2020 [consultado 12 septiembre 2020]1-35 Disponible en: https://bit.ly/3tZFlyE

28. Rocha Formiga F, Leblanc R, Reboucas JdS, Paiva Farias L, de Oliveria RN, Pena L. Ivermectin: an award winning drug with expected antiviral activity against COVID-19. J Control Release [Internet]. 2020 [consultado 14 septiembre 2020];329:758-761 Disponible en: https://www.arca.fiocruz. br/bitstream/icict/44006/4/Formiga_Fabio_etal_IGM_2020_COVID-19.pdf

29. Horby $P$, Lin WS, Emberson JR, Mafhan $\bar{M}$, Beli JL, Linsell L, et al. Dexamethasone in hospitalized patients with COVID-19: preliminary report. N Engl J Med [Internet]. 2020 [consultado 9 marzo 2021];384(8):693-704. Disponible en: https://www.ncbi.nlm.nih.gov/pmc/articles/PMC7383595/pdf/ NEJMoa2021436.pdf

30. Tomazini BM, Maia IS, Cavalcanti AB, Berwanger O, Rosa RG, Veiga VC, et al. Effect of Dexamethasone on days alive and ventilator-free in patients with moderate or severe acute respiratory distress syndrome and COVID-19. JAMA [Internet]. 2020 [consultado 9 marzo 2021];324(13):1307-1316. Disponible en: https://pubmed.ncbi.nlm.nih.gov/32876695/

31. Wu Y, Hou B, Liu J, Chen Y, Zhong P. Risk factors associated with long-term hospitalization in patients with COVID-19: a single-centered, retrospective study. Front Med (Lausanne) [Internet]. 2020 [consultado 17 septiembre 2020];7:315. Disponible en: https://www.ncbi.nlm.nih.gov/pmc/articles/ PMC7296106/

32. Servín Torres E, Nava Leyva H, Romero García A, Sánchez González F, Huerta García G. Equipo de protección personal y COVID-19. Cir Gen [Internet]. 2020 [consultado 14 septiembre 2020];42(2):116-123. Disponible en: https://www.medigraphic.com/cgi-bin/new/resumen. cgi?IDARTICULO=95370

33. Sánchez-Villena AR, de La Fuente-Figuerola V. COVID-19: Cuarentena, distanciamiento social, y confinamiento ¿son lo mismo? An Pediatr [Internet]. 2020 [consultado 23 diciembre 2020];93(1):73-74. Disponible en: https://www.analesdepediatria.org/es-covid-19-cuarentena-aislamientodistanciamiento-social-articulo-S1695403320301776

ABSTRACT. The purpose of this review was to analyze the existing literature in order to identify the main aspects of the disease caused by SARS-CoV-2, as well as its impact on the mental health of the elderly and its relationship with mortality in this population at risk. The information from articles in electronic journals was reviewed through the Pubmed, Medline, Scopus and Google Scholar databases for the period July 2020 to June 2021. Among the relevant aspects, alarming data were found, patients with comorbidities in this age group were 6 times more likely to be hospitalized and 12 times more likely to die than a person without comorbidities. Mental health is an important factor in this pandemic, deterioration in general condition and mental illnesses due to anxiety and social isolation secondary to the pandemic have been demonstrated in this group.

Keywords: Coronavirus Infections; Elderly; Mental Health. 